\title{
Usefulness of random-start progestin- primed ovarian stimulation for fertility preservation
}

Haipeng Huang* ${ }^{*}$, Yukiko Itaya, Kouki Samejima, Shunichiro Ichinose, Tatsuya Narita, Shigetaka Matsunaga, Masahiro Saitoh and Yasushi Takai

\begin{abstract}
Background: Progestin-primed ovarian stimulation (PPOS) has been used in infertility cases in recent years, and several reports have stated that it has oocyte collection results similar to those of gonadotropin-releasing hormone antagonist (GnRH-ant) protocol. For emergency fertility preservation, random-start ovarian stimulation is usually recommended. Therefore we compared the clinical outcomes of random-start PPOS with those of conventional randomstart $\mathrm{GnRH}$-ant protocols in fertility-preserving cases.

Methods: We retrospectively examined 86 cycles of oocyte collection, of which 56 were random-start GnRH-ant and 30 were random-start PPOS for fertility preservation at our hospital between January 2016 and April 2021. The primary outcome was the number of mature oocytes per cycle. The secondary outcome was the number of vitrified blastocysts per cycle for embryo freezing cases.

Results: No significant differences were noted in the number of days of stimulation, total dose of gonadotropin preparation, and the number of mature oocytes and vitrified blastocysts. The number of hospital visits for monitoring was significantly lower in the PPOS group. The start of menstruation before oocyte collection was significantly less in the PPOS group.

Conclusions: Random-start PPOS and GnRH-ant were similar in oocyte collection results. PPOS can reduce the number of hospital visits, thus reducing patient stress. PPOS at the start of the luteal phase can prevent the start of menstruation during ovarian stimulation.
\end{abstract}

Keywords: Breast cancer, Dydrogesterone, Fertility preservation, Letrozole, Ovarian stimulation

\section{Background}

In fertility-preserving treatment, oocytes should be collected within 2 to 3 weeks after the patient examination to avoid delaying the start of treatment for the underlying disease [1]. Therefore it is better to start ovarian stimulation as soon as possible unless its yield is compromised.

${ }^{*}$ Correspondence: haipeng3@saitama-med.ac.jp

Department of Obstetrics and Gynecology, Saitama Medical Center, Saitama Medical University, 1981 Kamoda, Kawagoe, Saitama 350-3550, Japan
Mounting evidence indicates that follicle development may occur 2 to 3 times in one menstrual cycle [2], and in 2013, Cakmak et al. reported the efficacy of a random-start gonadotropin releasing-hormone antagonist (GnRH-ant) protocol [3], which became the common ovarian stimulation technique in fertility-preserving cases. In a systematic review in 2021 [4], a conventional method that started stimulation from the early follicular phase $(n=1012)$ was compared with a random-start method that started stimulation from the late follicular and luteal phases $(n=641)$. Although not significantly original author(s) and the source, provide a link to the Creative Commons licence, and indicate if changes were made. The images or other third party material in this article are included in the article's Creative Commons licence, unless indicated otherwise in a credit line to the material. If material is not included in the article's Creative Commons licence and your intended use is not permitted by statutory regulation or exceeds the permitted use, you will need to obtain permission directly from the copyright holder. To view a copy of this licence, visit http://creativecommons.org/licenses/by/4.0/. The Creative Commons Public Domain Dedication waiver (http://creativeco mmons.org/publicdomain/zero/1.0/) applies to the data made available in this article, unless otherwise stated in a credit line to the data. 
different, the random-start method had a longer stimulation period (10.4 vs. 10.1 days, respectively) and higher total gonadotropin usage ( 2688 vs. $2575 \mathrm{IU}$, respectively) on average than those of the conventional method. Furthermore, although the number of mature oocytes collected was similar, the estradiol (E2) levels at the time of trigger were significantly lower in the random-start method on average than in the conventional method $(1128$ vs. $1855 \mathrm{pg} / \mathrm{mL}$, respectively), reconfirming the safety of its use in fertility-preserving cases.

In contrast, progestin-primed ovarian stimulation (PPOS) has been used in infertility cases in recent years, and it was shown to prevent unexpected increases in luteinizing hormone (LH) more efficiently than GnRH-ant. PPOS is also more affordable, and several reports have stated that it has oocyte collection results similar to that of GnRH-ant $[4,5]$. We believe that PPOS is suitable for the suppression of unexpected LH surges as a randomstart method for fertility-preserving cases with sustained gonadotropin suppression due to negative feedback from progestins. Therefore, we conducted a comparative study between the random-start PPOS and GnRH-ant methods. This study is the first to show the usefulness of random-start PPOS in fertility-preserving cases.

\section{Materials and method}

\section{Study setting and patients}

We retrospectively examined 86 cycles of oocyte collection for fertility preservation at our hospital between January 2016 and April 2021. Breast cancer cases accounted for $73 \%$ of the cases, followed by hematological disorders at $15 \%$. For patients with male partners either with or without legal marriage, oocyte and/or embryo cryopreservation were offered for fertility preservation. For patients without male partners, only oocyte cryopreservation was offered. Semen analysis for male partners was always performed before oocyte collection in couples with no history of pregnancy. The cases in this study did not have severe male infertility. The endpoints were the number of days of stimulation, total dose of gonadotropin, serum E2 concentrations on the day of ovulation trigger, oocyte maturation rate, fertilization rate in cases of embryo freezing, and good blastocyst rate. This research protocol was approved by the Saitama Medical Center, Saitama Medical University Ethics Review Board (approval number: 2575). This study was not funded, and it was performed retrospectively using electronic medical record information and ultrasound findings.

\section{Controlled ovarian stimulation}

For referred fertility-preserving cases, ovarian stimulation was promptly started regardless of the menstrual cycle if oocyte collection was desired after counseling.
A random-start PPOS or GnRH-ant protocol was used depending on the practice of the attending physicians and the patient preference of ovarian stimulation method.

Both methods were initiated by subcutaneous injection of recombinant FSH (GONAL-f; Merck). All of the breast cancer patients were referred to our hospital along with the information of estrogen receptor status which had been tested either on the biopsy sample or on the surgical specimen. For estrogen receptor-positive breast cancer cases, $5 \mathrm{mg} /$ day of letrozole was used in combination from the start of ovarian stimulation to the day of trigger.

In the $\mathrm{GnRH}$-ant group, a flexible method was adopted wherein $0.25 \mathrm{mg}$ of ganirelix acetate (Ganirest, MSD) was administered daily from the day when the main follicle diameter reached $14 \mathrm{~mm}$ by ovarian stimulation. Follicular diameter was defined as the average value of the major and minor axes. In contrast, in the PPOS group, $20 \mathrm{mg}$ of dydrogesterone (Duphaston, Mylan) was administered each day from the start of ovarian stimulation to the day of trigger (Fig. 1). In both groups, follicular growth was monitored every 2-5 days using serum levels of FSH, $\mathrm{LH}, \mathrm{E} 2$, and progesterone (P4), as well as transvaginal ultrasonography.

In both groups without letrozole, in general, when it was confirmed that there were $\geq 2$ follicles of $\geq 18$-mm diameter, dual trigger with four nasal sprays $(150 \mu \mathrm{g}$ each $)$ of buserelin (Buserelin, Fuji) and an injection of $1500 \mathrm{IU}$ of urine human chorionic gonadotropin (HCG) (HCG, Fuji) was administered to induce maturation of oocytes, and oocyte pickup was performed $35-37 \mathrm{~h}$ later. In the groups with letrozole, however, the dual trigger was delayed until when there were $\geq 1$ follicles of $\geq 20$-mm diameter. Also, even when the sizes of dominant follicles reach 18 or $20 \mathrm{~mm}$, the time of triggering may be slightly delayed when the sizes of other nondominant follicles do not reach $16 \mathrm{~mm}$ in order to maximize the number of maturate oocytes.

\section{Embryo culture and assessment}

Patients with male partners who opted for embryo cryopreservation underwent intracytoplasmic sperm injection (ICSI), in vitro fertilization (IVF), or split insemination after oocyte collection. Although selection was based mainly on the preference of the patient, ICSI was actively recommended in cases with poor semen findings on the day of oocyte collection. The cases in this study did not include severe male infertility.

\section{ICSI}

On day 0 of oocyte retrieval, the collected eggs were pipetted to remove the granulosa cell layer (PINU0620FT, Prime Tech Ltd.) connected to a piezo-electric actuator (Prime Tech Ltd.). After injecting sperm, the 


\section{0:30 HCG 1500 IU}

22:00 \& 23:00 $300 \mu \mathrm{g}$ of Buserelin

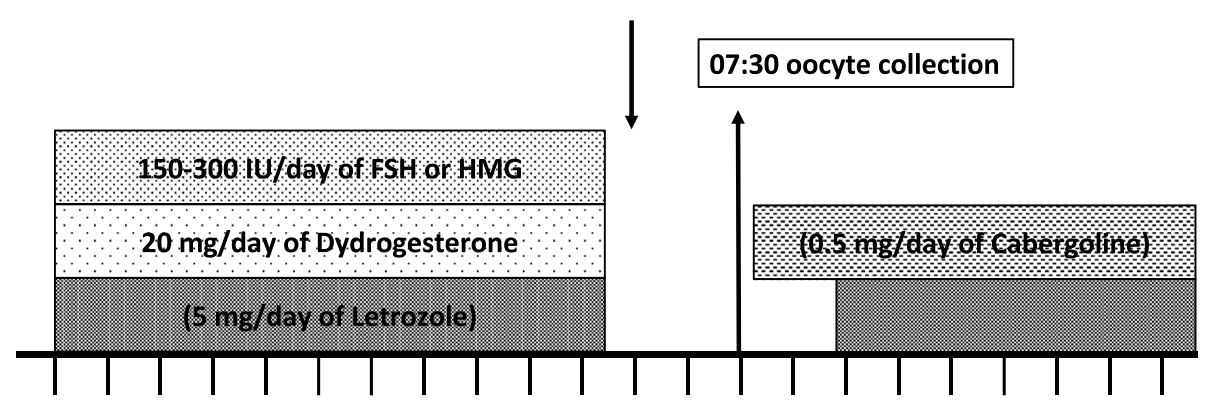

Fig. 1 Random-start progestin-primed ovarian stimulation regimens. Abbreviations: FSH, follicle-stimulating hormone; HMG, human menopausal gonadotropin; HCG, human chorionic gonadotropin

cells were cultured using a single-step culture medium (SAGE 1-Step, CooperSurgical). Embryos were observed in the time-lapse culture system (Astec Co. Ltd.) on days $1,2,3,5$, and 6 after oocyte retrieval. Normal fertilization was determined if two pronuclei were observed on day 1 after oocyte retrieval. Blastocysts were evaluated according to the blastocyst scoring system developed by Gardner et al. [6], and blastocysts that reached Grade 3BB were frozen.

\section{IVF}

Within $2 \mathrm{~h}$ of oocyte collection, the final sperm concentration was adjusted to $300,000 / \mathrm{mL}$, and insemination was performed. Fertilization was determined when the granulosa cell layer was removed, and the second polar body was confirmed approximately $5 \mathrm{~h}$ after fertilization.

\section{Study outcomes}

The primary outcome was numbers of oocytes and metaphase two oocytes per cycle. The secondary outcome was the number of vitrified blastocysts per cycle for embryo freezing cases. In addition, AMH levels, ovarian stimulation period, total dose of gonadotropin preparation, days of hospital visits, start of menstruation rate before oocyte collection, number of collected oocytes, number of mature oocytes, and number of normal fertilized oocytes were comparatively examined. Oocyte maturation was evaluated at the timing of oocyte vitrification and ICSI. For the oocytes with which standard IVF was performed, oocyte maturation was evaluated under the removal of cumulus cells $4 \mathrm{~h}$ after insemination.

\section{Statistical analysis}

All statistical analyses were performed using JMP version 16.0.0 software. Patient characteristics, ovarian stimulation characteristics, and endocrinological characteristics were expressed as mean ( \pm standard deviation). For frequency/ratio comparisons, chi-squared test with Yates' correction or Fisher's exact test and the average ratio were used. The Student's t-test was used for comparison. Statistical significance was set at $P<0.05$.

\section{Results}

\section{Patient characteristics}

Table 1 compares the characteristics of the patients. There were no significant differences in age, history of pregnancy, the presence or absence of male partner, BMI, serum AMH levels, and the timing of ovarian stimulation (follicular or luteal phase). There were no patient with history of infertility or polycystic ovary syndrome in the present study.

\section{Outcomes of ovarian stimulation}

Table 2 shows the outcomes of ovarian stimulation in both groups. The total amount of gonadotropin used, administration periods, number of eggs collected, and maturation rates of eggs were similar. No cases were canceled due to unexpected side effects in either group. The start of menstruation before oocyte collection was significantly lower in the PPOS group than in the

Table 1 Comparison of patient characteristics between random-start GnRH-ant and random-start PPOS groups

\begin{tabular}{llll}
\hline & GnRH-ant, $\boldsymbol{n}=\mathbf{5 6}$ & PPOS, $\boldsymbol{n}=\mathbf{3 0}$ & $\boldsymbol{P}$ value \\
\hline Age, years & $32.6(0.9)$ & $31.7(1.2)$ & 0.531 \\
Primigravida, \% & $75.0 \%$ & $73.3 \%$ & 0.865 \\
No male partner, \% & $66.1 \%$ & $53.4 \%$ & 0.256 \\
BMl & $21.8(0.5)$ & $20.9(0.6)$ & 0.288 \\
AMH, ng/mL & $2.76(0.37)$ & $3.47(0.51)$ & 0.268 \\
Luteal phase start, \% & 41.8 & 22.6 & 0.099 \\
\hline
\end{tabular}

Values are expressed as the mean ( \pm standard deviation) 
Table 2 Comparison of oocyte collection results

\begin{tabular}{|c|c|c|c|}
\hline & $\begin{array}{l}\text { GnRH-ant, } n=56 \\
\text { (Letrozole combination, } n=38 \text { ) }\end{array}$ & $\begin{array}{l}\text { PPOS, } n=30 \\
\text { (Letrozole combination, } n=18 \text { ) }\end{array}$ & $P$ value \\
\hline HMG dose & $2527.6(73.3)$ & $2512.9(100.2)$ & 0.905 \\
\hline HMG duration, days & $11.0(0.3)$ & $11.2(0.4)$ & 0.616 \\
\hline Number of collected oocytes & $9.9(0.9)$ & $12.2(1.2)$ & 0.137 \\
\hline Number of mature oocytes & $8.4(0.8)$ & $10.8(1.1)$ & 0.099 \\
\hline Maturation rate, \% & $84.1(2.4)$ & $89.5(3.3)$ & 0.195 \\
\hline Number of visits for monitoring & $4.2(0.1)$ & $3.4(0.14)$ & $<0.001^{*}$ \\
\hline Start of menstruation before oocyte collection, $\%$ & 25.0 & 3.3 & $0.013^{*}$ \\
\hline
\end{tabular}

Values are expressed as the mean ( \pm standard deviation). Significant $P$ values are presented in bold and denoted with an asterix

Abbreviations: GnRH-ant Gonadotropin-releasing hormone antagonist, HMG Human menopausal gonadotropin, $L H$ Luteinizing hormone, $P 4$ Progesterone, PPOS Progestin-primed ovarian stimulation

Table 3 Comparison of frozen embryo cases

\begin{tabular}{llll}
\hline & $\begin{array}{l}\text { GnRH-ant } \\
\boldsymbol{n}=\mathbf{3 7} \\
\text { (Letrozole } \\
\text { combination, } \\
\boldsymbol{n = 2 7 )}\end{array}$ & $\begin{array}{l}\text { PPOS, } \boldsymbol{n}=\mathbf{1 6} \\
\text { (Letrozole } \\
\text { combination, } \\
\boldsymbol{n}=\mathbf{1 0} \text { ) }\end{array}$ & P value \\
\hline HMG dose & $2552.7(97.3)$ & $2438.2(148.1)$ & 0.521 \\
HMG duration, days & $11.1(0.3)$ & $10.9(0.5)$ & 0.658 \\
Number of collected & $9.8(1.0)$ & $10.3(1.5)$ & 0.779 \\
oocytes & $89.0(2.4)$ & $90.1(3.7)$ & 0.807 \\
Maturation rate, \% & 78.4 & 87.5 & 0.704 \\
ICSI rate, \% & $67.9(4.0)$ & $64.6(6.1)$ & 0.655 \\
Fertilization rate, \% & $2.8(0.5)$ & $2.3(0.7)$ & 0.551 \\
$\begin{array}{l}\text { Number of frozen blas- } \\
\text { tocysts }\end{array}$ & & $61.3(8.1)$ & 0.872 \\
Good blastocyst rate, \% & $59.8(5.4)$ & &
\end{tabular}

Values are expressed as the mean ( \pm standard deviation)

Abbreviations: GnRH-ant Gonadotropin-releasing hormone antagonist, HMG Human menopausal gonadotropin, LH Luteinizing hormone, P4 Progesterone, PPOS Progestin-primed ovarian stimulation

GnRH-ant group (3.3\% vs. $25.0 \%$, respectively; $P=0.011$ ). The number of hospital visits for monitoring was significantly lower in the PPOS group than in the GnRHant group ( 3.4 vs. 4.1 , respectively; $P<0.001$ ). When the comparison of visits was performed separately for the follicular-phase stimulation cases and for the lutealphase stimulation ones, the number of visits in PPOS was still significantly lower than that in GnRH-ant (data not shown).

\section{Embryological outcomes}

Thirty-seven patients in the GnRH-ant group and sixteen in the PPOS group chose to freeze blastocyst. A comparison of the results is presented in Table 3. The fertilization rates, number of frozen blastocysts, and blastocyst rates were similar.

\section{Aromatase inhibitors}

Thirty-eight patients were included in the GnRH-ant group and eighteen patients in the PPOS group. The letrozole combination was administered in estrogen receptor-positive breast cancer cases, and significantly suppressed serum E2 levels on trigger day, but the number of stimulation days, amount of gonadotropin used, number of eggs collected, and mature egg rates were not affected. In addition, the fertilization rates, number of frozen blastocysts, and blastocyst rates were similar (data not shown).

\section{Discussion}

This study is the first to show the usefulness of random-start PPOS in fertility-preserving cases. We found that there was no difference in oocyte collection results between the random-start GnRH-ant and PPOS methods. In addition, since progestin was continuously administered in PPOS, random-start PPOS significantly suppressed the start of menstruation before oocyte collection compared with random-start GnRH-ant method.

Uterine bleeding can be a major problem in hematological disorder cases with thrombocytopenia, and also for other cancer patients without thrombocytopenia, it may be an advantage of random-start PPOS that the appearance of menstruation does not bother patients and caregivers during ovarian stimulation and oocyte collection. As shown in the present study, moreover, PPOS can reduce the number of hospital visits for monitoring since it was reported to be more efficient at preventing LH surge than GnRH-ant protocol [7] and it is enough to start monitoring 1 week after the start of PPOS, while in $\mathrm{GnRH}$-ant protocol monitoring should be started earlier and be more frequent to prepare for unexpected $\mathrm{LH}$ rise. Thus PPOS may reduce patients' stress and gain an advantage in the cost-effectiveness together with the low cost of progestins. 
Since the first report by Kuang et al. in 2015 [8], PPOS has been expected to perform as well as the GnRHant method, and there is consensus that it is an affordable and useful ovarian stimulation method with a low cancelation rate. Previous studies on PPOS have compared the GnRH-ant protocol with PPOS [4, 5, 9-11], and the target patients were mainly normal responders.

The effect of progesterone on oocyte quality has also been investigated. In the random-start GnRH-ant method, 238 cases that underwent freeze-all strategy were divided into two groups based on P4 concentrations at the time of trigger $(<1.5 \mathrm{ng} / \mathrm{mL}$ and $\geq 1.5 \mathrm{ng} /$ $\mathrm{mL}$ ). There were no significant differences in embryonic aneuploidy [12]. There is also a report on preimplantation genetic testing for aneuploidies (PGT-A) of PPOS [5] where PGT-A was performed on 785 blastocysts obtained by an age-matched GnRH-ant method or PPOS at 37 years of age. The results revealed similar rates for euploid embryos in both groups. Within this context, this could become the basis for providing random-start PPOS to fertility-preserving cases without concerns.

Breast cancer is the most common disease for which assisted reproductive technology is performed for fertility preservation. For hormone-dependent tumors, it is ideal to use letrozole, an aromatase inhibitor, to avoid an increase in serum E2 concentrations [13]. However, a meta-analysis recently confirmed that menopausal hormone replacement therapy containing progestins such as (levo) norgestrel, norethisterone acetate, and medroxyprogesterone acetate increases the risk of breast cancer, compared to never users or estrogen-only users [14]. We assume that PPOS with dydrogesterone may be relatively safe because the above-mentioned meta-analysis showed that its use less than 5 years had been no effect on the risk of breast cancer, but the safety of PPOS for breast cancer patients should be carefully elucidated in the future studies. We decided the dosage of dydrogesterone according to the previous study [15], in which MPA $10 \mathrm{mg} / \mathrm{d}$ is too strong for LH suppression compared with dydrogesterone $20 \mathrm{mg} / \mathrm{d}$. Since we sometimes decrease the dosage of dydrogesterone to $10 \mathrm{mg} / \mathrm{d}$ when the serum $\mathrm{LH}$ level is suppressed below $1.0 \mathrm{mIU} / \mathrm{ml}$, we assume that the optimization or individualization of progestin dosage is necessary, especially for breast cancer cases.

The limitation of this study is that it was a retrospective study with a small number of cases, and that it was a single center study and that most participants had breast cancer. By accumulating random-start PPOS cases with hematological symptoms such as thrombocytopenia, we expect future studies to investigate the possibility of reducing the risk complications in oocyte collection during menstruation. We also believe that patients who have overcome cancer treatment would want to know the pregnancy results when using collected oocytes and frozen embryos.

Random-start PPOS is a simpler and more affordable ovarian stimulation method than random-start GnRH-ant. Both methods yielded similar oocyte collection results. Moreover, PPOS in luteal phase can prevent the start of menstruation during ovarian stimulation and is considered to be particularly useful in cases with thrombocytopenia.

\section{Abbreviations \\ AMH: Anti-Mullerian hormone; BMl: Body mass index; FSH: Follicle-stimulating hormone; GnRH-ant: Gonadotropin releasing-hormone antagonist; HCG: Human chorionic gonadotropin; ICSI: Intracytoplasmic sperm injection; IVF: In vitro fertilization; LH: Luteinizing hormone; PGT-A: Preimplantation genetic testing for aneuploidies; PPOS: Progestin-primed ovarian stimulation.}

\section{Acknowledgements}

We would like to thank Editage for English language editing.

\section{Authors' contributions}

$\mathrm{HH}$ was a major contributor in writing the manuscript. YT was involved in drafting and revising the manuscript. KS, TN, SI, SM and MS were involved in revising the manuscript. YI contributed to the study concept and design. All the authors read and approved the final manuscript.

\section{Funding}

This report received no specific grant from any funding agency in the public, commercial, or not-for-profit sectors.

Availability of data and materials

Not applicable.

\section{Declarations}

\section{Ethics approval and consent to participate}

The study protocol was approved by the ethics committees of Saitama Medical Center of Saitama Medical University (approval no. 2575). The requirement for consent for participation was waived due to the retrospective nature of the study.

Consent for publication

Not applicable.

\section{Competing interests}

The authors declare that that they have no competing interests.

Received: 10 July 2021 Accepted: 11 December 2021

Published online: 04 January 2022

\section{References}

1. Danis RB, Pereira N, Elias RT. Random start ovarian stimulation for oocyte or embryo cryopreservation in women desiring fertility preservation prior to Gonadotoxic Cancer therapy. Curr Pharm Biotechnol. 2017;18(8):609-13.

2. Baerwald AR, Adams GP, Pierson RA. Ovarian antral folliculogenesis during the human menstrual cycle: a review. Hum Reprod Update. 2012;18:73-91.

3. Cakmak H, Katz A, Cedars MI, Rosen MP. Effective method for emergency fertility preservation: random-start controlled ovarian stimulation. Fertil Steril. 2013;100:1673-80.

4. Gurbuz AS, Gode F. Dydrogesterone-primed ovarian stimulation is an effective alternative to gonadotropin-releasing hormone antagonist 
protocol for freeze-all cycles in polycystic ovary syndrome. J Obstet Gynaecol Res. 2020;46:1403-11.

5. La Marca A, Capuzzo M, Sacchi S, Imbrogno MG, Spinella F, Varricchio MT, et al. Comparison of euploidy rates of blastocysts in women treated with progestins or $\mathrm{GnRH}$ antagonist to prevent the luteinizing hormone surge during ovarian stimulation. Hum Reprod. 2020;35:1325-31.

6. Gardner DK, Lane M, Stevens J, Schlenker T, Schoolcraft WB. Blastocyst score affects implantation and pregnancy outcome: towards a single blastocyst transfer. Fertil Steril. 2000;73:1155-8.

7. Mathieu d'Argent E, Ferrier C, Zacharopoulou C, Ahdad-Yata N, Boudy AS, Cantalloube A, et al. Outcomes of fertility preservation in women with endometriosis: comparison of progestin-primed ovarian stimulation versus antagonist protocols. J Ovarian Res. 2020;13:18.

8. Kuang Y, Chen Q, Fu Y, Wang Y, Hong Q, Lyu Q, et al. Medroxyprogesterone acetate is an effective oral alternative for preventing premature luteinizing hormone surges in women undergoing controlled ovarian hyperstimulation for in vitro fertilization. Fertil Steril. 2015;104:62-70.

9. Chen Q, Chai W, Wang Y, Cai R, Zhang S, Lu X, Zeng X, Sun L, Kuang Y. Progestin vs. gonadotropin-releasing hormone antagonist for the prevention of premature luteinizing hormone surges in poor responders undergoing in vitro fertilization treatment: a randomized controlled trial. Front Endocrinol (Lausanne) 2019;10:796.

10. Yildiz S, Turkgeldi E, Angun B, Eraslan A, Urman B, Ata B. Comparison of a novel flexible progestin primed ovarian stimulation protocol and the flexible gonadotropin-releasing hormone antagonist protocol for assisted reproductive technology. Fertil Steril. 2019;112:677-83.

11. Iwami N, Kawamata M, Ozawa N, Yamamoto T, Watanabe E, Moriwaka O, et al. New trial of progestin-primed ovarian stimulation using dydrogesterone versus a typical $\mathrm{GnRH}$ antagonist regimen in assisted reproductive technology. Arch Gynecol Obstet. 2018;298:663-71.

12. Kofinas JD, Mehr H, Ganguly N, Biley Y, Bochkovsky S, McCulloh D, et al. Is it the egg or the endometrium? Elevated progesterone on day of trigger is not associated with embryo ploidy nor decreased success rates in subsequent embryo transfer cycles. J Assist Reprod Genet. 2016;33:1 169-74.

13. Kasum $M$, von Wolff M, Franulic $D$, Čehić E, Klepac-Pulanić T, Orešković $\mathrm{S}$, et al. Fertility preservation options in breast cancer patients. Gynecol Endocrinol. 2015;31:846-51.

14. Collaborative Group on Hormonal Factors in Breast Cancer. Type and timing of menopausal hormone therapy and breast cancer risk: individual participant meta-analysis of the worldwide epidemiological evidence. Lancet. 2019;394:1159-68.

15. Yu S, Long H, Chang HY, Liu Y, Gao H, Zhu J, et al. New application of dydrogesterone as a part of a progestin-primed ovarian stimulation protocol for IVF: a randomized controlled trial including 516 first IVF/ICSI cycles. Hum Reprod. 2018;33:229-37.

\section{Publisher's Note}

Springer Nature remains neutral with regard to jurisdictional claims in published maps and institutional affiliations.

Ready to submit your research? Choose BMC and benefit from:

- fast, convenient online submission

- thorough peer review by experienced researchers in your field

- rapid publication on acceptance

- support for research data, including large and complex data types

- gold Open Access which fosters wider collaboration and increased citations

- maximum visibility for your research: over $100 \mathrm{M}$ website views per year

At BMC, research is always in progress.

Learn more biomedcentral.com/submissions 